\title{
BMJ Open Impact of internal carotid endarterectomy on visual fields: a non- randomised prospective cohort study in Austria
}

Peter Konstantiniuk, ${ }^{1}$ Iris Steinbrugger, ${ }^{2}$ Stephan Koter, ${ }^{1}$ Johanna Muehlsteiner, ${ }^{1}$ Andreas Wedrich, ${ }^{2}$ Tina Cohnert ${ }^{1}$

To cite: Konstantiniuk $P$, Steinbrugger I, Koter S, et al. Impact of internal carotid endarterectomy on visual fields: a non-randomised prospective cohort study in Austria. BMJ Open 2017;7:e017027. doi:10.1136/ bmjopen-2017-017027

- Prepublication history for this paper is available online. To view these files, please visit the journal online (http://dx.doi. org/10.1136/bmjopen-2017017027).

Received 27 March 2017 Revised 1 September 2017 Accepted 5 September 2017

CrossMark

${ }^{1}$ Department of Surgery, Division of Vascular Surgery, Medical University of Graz, Graz, Steiermark, Austria

${ }^{2}$ Department of Ophthalmology, Medical University of Graz, Graz, Steiermark, Austria

Correspondence to Dr Peter Konstantiniuk; peter.konstantiniuk@ medunigraz.at

\section{ABSTRACT}

Purpose The goal of this study was to determine whether internal carotid endarterectomy is associated with visual field changes.

Methods Between March 2007 and December 2010, a cohort study involved 29 patients with stenosis of the carotid artery. All patients underwent ophthalmoscopy and kinetic visual field examination (Goldmann perimetry) preoperatively and postoperatively. Furthermore, a detailed area calculation was performed. On both the operated and the contralateral side, the areas surrounded by the different isopters (isopter areas) were determined and preoperative and postoperative values compared. The three isopters were classified from the centre to the periphery (I, II and III). Isopter area values are given as ratio compared with total perimetric circle. $p$ Values $<0.05$ were considered to be statistically significant.

Results 1) The ophthalmologists assessment Eight of eleven patients with precarotid endarterectomy impairments experienced focal or concentric improvement. Six cases with preoperative concentric narrowing of the isopters returned to normal or improved substantially. Three out of 18 patients with normal preoperative visual field presented with deteriorations after surgery, two cases with ipsilateral and one with contralateral focal impairment. In 15 cases, there was no preoperative or postoperative abnormality. 2) Area calculation (preoperative area, postoperative area, P) Ipsilateral: isopter area I $(0.015,0.018,0.131)$, isopter area II $(0.107,0.120,0.087)$, isopter area III $(0.392,0.425$, $0.015)$ Contralateral: isopter area I $(0.017,0.021,0.222)$, isopter area II $(0.119,0.125,0.333)$, isopter area III $(0.416$, $0.434,0.171)$

Conclusions We found a statistically significant extension of the ipsilateral peripheral isopter area (III). Further studies will focus on the question which subgroup is most likely to profit from internal carotid endarterectomy with respect to visual field changes.

\section{INTRODUCTION}

Large trials have proven internal carotid endarterectomy (ICE) to be effective in reducing the risk of stroke in patients with internal carotid artery stenosis ${ }^{1-7}$. Ocular

\section{Strengths and limitations of this study}

Kinetic visual field examination (Goldmann) was used which is not affected by learning effects.

- The window between preoperative and postoperative examination was small ( $\leq 9$ days) to avoid biasing by other factors.

- The study sample was too small to identify influencing factors.

- The postoperative visual field examination was performed within 48 hours after surgery. Some patients were weak and had difficulties to concentrate. Therefore, a postponement of this examination could have improved the postoperative results.

- It is unclear, if the postoperatively determined visual field improvements are persistent in the long run.

events were used as outcome parameters in some trials, ${ }^{2} 7$ whereas in others they were only mentioned as baseline characteristics. This is somewhat inconsistent since both one layer of the retina and the optical nerve are morphologically parts of the brain. Thus retinal infarction, amaurosis fugax and optic neuropathy ${ }^{8}$ are nothing but very specific subtypes of stroke. All these changes can be quantified on a functional level with visual field examinations. We know from peripheral artery disease (PAD) and from coronary artery disease (CAD) that revascularisation is associated with functional improvements. These can be measured as walking distance with a treadmill for PAD and as ECG changes or shortness of breath with a cardiac stress test for CAD. ICE is performed to prevent stroke and not to improve cerebral as well as ocular perfusion. But all vascular surgeons know that there are sporadic patient reports of perioperative improvement of visual perception, thus we decided to investigate the correlation between carotid endarterectomy and the eye, 
or specifically, perioperative visual field changes. The goal was to determine whether there are any visual field changes at all and if so to determine the extent.

\section{MATERIALS AND METHODS}

From March until September 2007, a pilot study was conducted to reveal whether there are visual field changes with ICE or not. Based on these results, the sample size was extended (second recruitment period November 2008 until December 2010). Inclusion criteria for this cohort study were patients scheduled for ICE. Exclusion criteria were diseases with a high probability of visual field changes (diabetes mellitus, glaucoma), protocol deviations (preoperative visual field examination $>7$ days prior to ICE, postoperative visual field examination $>48$ hours after ICE), light of different size and/or brightness used for the preoperative versus the postoperative visual field examination. Patient selection was performed within organisational limitations. Asymptomatic patients were asked for participation, if Monday admission was possible to guarantee operation on Tuesday or Wednesday and have postoperative visual field measurement within 48 hours after surgery. Symptomatic patients were asked for participation, if postoperative visual field measurement was available within 48 hours after surgery. All patients gave informed consent.

All patients underwent ophthalmoscopy and kinetic visual field examination (Goldmann-Perimeter, Haag-Streit, Bern, Switzerland). The latter uses mobile light of defined and constant size and brightness moved by an examiner from the periphery towards the centre of vision until it is first detected by the patient. This is repeated by approaching the centre of vision from different directions. The examiner marks the threshold of visibility on a purpose-made documentation sheet. Points of equal visual sensitivity can be connected and are then called isopters. The procedure is repeated using different test lights that are larger or brighter than the original one. Both ophthalmoscopy and analysis of the visual field examination were performed by an ophthalmologist. Neither the examiner nor the ophthalmologist were blinded for the side of operation.

The first ophthalmological investigation was performed 1-7 days before surgery, the second, 24-48 hours after surgery. The three isopters were classified from the centre to the periphery (I, II and III). Furthermore, the areas surrounded by the different isopters were determined by sectioning the area into triangles with a central angle of $15^{\circ}$ and adding them up. Isopter area II includes area I, isopter area III includes area I and II. Isopter area values are given in ratio compared with the total perimetric circle. Each patient had the same test light preoperatively and postoperatively.

Preoperative and postoperative values were compared with SPSS V.23.0.0.0. Since preoperative and postoperative isopter areas are ratios they were transformed using the logit function $\operatorname{logit}(\mathrm{x})=\ln (\mathrm{x} / 1-\mathrm{x})$. The transformed values were compared with a paired samples t-test. Mean values and CIs were calculated from the transformed values and then retransformed using the function inverse-logit $(\mathrm{x})=\exp (\mathrm{x}) /(\exp (\mathrm{x})+1)$. Analogously, we investigated the following factors of potential impact on isopter area III changes:

a) contralateral internal carotid artery (ICA) occlusion (comparison with t-test);

b) grade of ipsilateral ICA stenosis (up to $80 \%$ was scored with $1,>80 \%$ with 2 , comparison with t-test);

c) residual blood flow (the listed vessels were included in a score, 2 points for not obstructed vessels, 1 point for a vessel with significant stenosis, no point for an occluded vessel: contralateral ICA, ipsilateral and contralateral external carotid artery, ipsilateral and contralateral vertebral artery, statistical evaluation with regression analysis);

d) clinical status of the patient (symptomatic vs asymptomatic, comparison with t-test).

p Values $<0.05$ were considered to be statistically significant.

\section{Study size}

This study was performed in two stages. First, a pilot study was undertaken with 12 patients. Power calculation on the area differences of these revealed 26 cases to achieve level of significance ( $\alpha$-error 0.05 , power 0.80 ).

\section{RESULTS}

\section{Patients characteristics}

During the pilot phase 14 patients gave informed consent. One patient was excluded for protocol violation (operation on a Friday thus no postoperative visual field examination within 48 hours), another patient was excluded for secondary not fulfilling the inclusion criteria (the patient was scheduled for ICE, but after admission the revascularisation technique was changed to carotid artery stenting due to a high tandem stenosis). During the second phase of the study, 22 patients were consented to participate. Two patients were excluded for protocol violation (no postoperative visual field examination within 48 hours), three were excluded for incomparable preoperative versus postoperative visual field examinations (light of different size and/or brightness). Patients who did not fulfil the inclusion criteria as well as those who were not prepared to consent were not registered. Thus, finally 20 non-consecutive patients with asymptomatic and 9 with symptomatic stenosis of the carotid artery were included in the study; $79.3 \%$ (23) were male and $20.7 \%$ (6) female. Mean age was 64.9 years (range 46.2-80.3, SD 9.1); $62.1 \%$ (18) of procedures were performed on the left and $37.9 \%$ (11) on the right side. The symptoms were: hypaesthesia in two cases, hypaesthesia in combination with partial aphasia, complete aphasia, visual field defects in two cases, occlusion of a branch of the retinal artery, amaurosis fugax and scintillating scotoma. Indication for surgery was based on an internal carotid artery stenosis of not $<70 \%$ (following NASCET criteria) diagnosed by duplex 
scan and nuclear magnetic resonance angiography. The latter was carried out to verify the ultrasound results and to exclude a severe tandem stenosis. All operations were performed under general anaesthesia. In 20 patients endarterectomy with patchplasty was done, 9 patients underwent eversion endarterectomy up to the surgeons' preference. The endarterectomy was expanded into the external carotid artery without seeking for completeness in all cases and without fixation of the cranial intimal flap. A shunt tube was used in general. Just prior to clamping of the ICA, 30 IU heparin per kg weight were administered intravenously. None of the patients developed new neurological deficits or experienced deterioration of pre-existing neurological symptoms.

\section{The ophthalmologists assessment}

The ipsilateral findings are displayed in detail in table 1 . Eight out of 11 patients with preoperative impairment improved after surgery. In three cases (\#3 , \#5, \#28figure 1), a preoperative concentric narrowing of the isopters disappeared postoperatively. In three other cases (\#19, \#23, \#25), a preoperative concentric narrowing presented with improvement postoperatively without achieving normal values. In two cases (\#22, \#26), a preoperatively focal deficit improved postoperatively. In two cases (\#10,\#11), a focal deficit was identified before operation and did not change perioperatively. One patient was found with contralateral temporal impairment preoperatively and postoperatively without change (\#4).

Three out of 18 patients with normal preoperative visual field presented with deteriorations after surgery. In one case (\#2, figure 2), we found a postoperative focal impairment of the ipsilateral eye. In one case (\#21), a preoperatively normal visual field presented with both focal improvements and impairments. Another patient presented with a contralateral temporal impairment (\#1). In 15 cases $(\# 6, \# 7, \# 8, \# 9, \# 12, \# 13, \# 14, \# 15, \# 16, \# 17$, \#18, \#20, \#24, \#27, \#29), there was no preoperative or postoperative abnormality.

\section{Area calculation}

Details are displayed in table 2. Isopter areas on both the operated and the contralateral side were larger postoperatively, but only isopter area III of the operated side showed a statistically significant difference $(\mathrm{p}=0.015)$. Isopter areas of the operated side were (not significantly) smaller compared with those of the contralateral eye. This difference was not observed in isopter areas II or III after surgery.

We performed a subgroup analysis of isopter area III of the operated side to assess risk factors for postsurgery differences (table 3 ). There were no statistically significant differences in the factors assessed.

\section{DISCUSSION}

In general, we found evidence that ICE is associated with a visual field improvement on the operated side. At the
Table 1 Preoperative and postoperative perimetric diagnosis of both the ipsilateral and the contralateral eye (NAD: no abnormality detected)

\begin{tabular}{|c|c|c|}
\hline No. & Preoperative & Postoperative \\
\hline 1 & NAD & $\begin{array}{l}\text { Contralateral temporal } \\
\text { impairment }\end{array}$ \\
\hline 2 & NAD & Ipsilateral temporal impairmen \\
\hline 3 & $\begin{array}{l}\text { Ipsilateral concentric } \\
\text { impairment }\end{array}$ & NAD \\
\hline 4 & $\begin{array}{l}\text { Contralateral temporal } \\
\text { impairment }\end{array}$ & $\begin{array}{l}\text { Contralateral temporal } \\
\text { impairment }\end{array}$ \\
\hline 5 & $\begin{array}{l}\text { Ipsilateral concentric } \\
\text { impairment }\end{array}$ & NAD \\
\hline 6 & NAD & NAD \\
\hline 7 & NAD & NAD \\
\hline 8 & NAD & NAD \\
\hline 9 & NAD & NAD \\
\hline 10 & Ipsilateral impairment frontal & Ipsilateral impairment frontal \\
\hline 11 & $\begin{array}{l}\text { Ipsilateral impairment } \\
\text { inferonasal }\end{array}$ & $\begin{array}{l}\text { Ipsilateral impairment } \\
\text { inferonasal }\end{array}$ \\
\hline 12 & NAD & NAD \\
\hline 13 & NAD & NAD \\
\hline 14 & NAD & NAD \\
\hline 15 & NAD & NAD \\
\hline 16 & NAD & NAD \\
\hline 17 & NAD & NAD \\
\hline 18 & NAD & NAD \\
\hline 19 & Ipsilateral concentric narrowing & Remarkable improvement \\
\hline 20 & NAD & NAD \\
\hline 21 & NAD & $\begin{array}{l}\text { Focal improvement in } \\
\text { combination with focal } \\
\text { impairment }\end{array}$ \\
\hline 22 & $\begin{array}{l}\text { Ipsilateral focal impairment } \\
\text { inferonasal }\end{array}$ & Minor improvement \\
\hline 23 & $\begin{array}{l}\text { Ipsilateral concentric } \\
\text { impairment }\end{array}$ & $\begin{array}{l}\text { Mainly normal with remaining } \\
\text { temporal impairment }\end{array}$ \\
\hline 24 & NAD & NAD \\
\hline 25 & $\begin{array}{l}\text { Ipsilateral concentric } \\
\text { impairment }\end{array}$ & Frontal improvement \\
\hline 26 & Ipsilateral frontal impairment & Minor improvement \\
\hline 27 & NAD & NAD \\
\hline 28 & $\begin{array}{l}\text { Ipsilateral concentric } \\
\text { impairment }\end{array}$ & NAD \\
\hline 29 & NAD & NAD \\
\hline
\end{tabular}

onset of our study, a very similar study was published. Kozobolis et a ${ }^{\ominus}$ performed visual field examinations preoperatively and postoperatively using the Octopus $500 \mathrm{EZ}$ automated perimeter. They found a visual field improvement reflected in significant differences in the 'mean defect' preoperatively versus postoperatively. These data are very consistent with our findings but determined with a completely different visual field measurement technique. Similar results were found by $\mathrm{Qu}$ et al. ${ }^{10}$ Like in the study of Kozobolis $e t$ al an automated static perimeter was used (Octopus 101). Their study population consisted of 


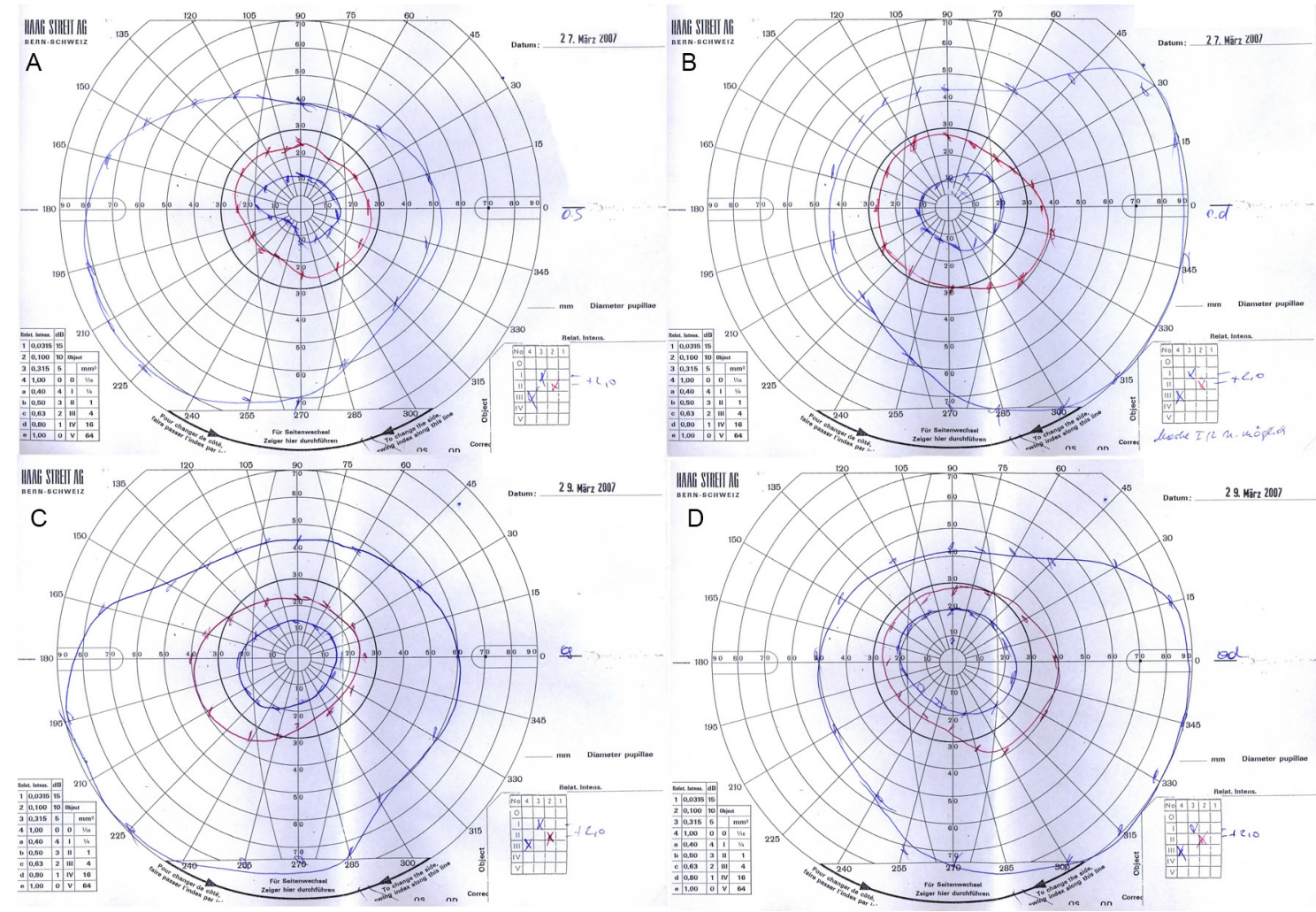

Figure 1 Preoperative and postoperative perimetry of patient \#3. Outer blue line: isopter III, red line: isopter II, inner blue line: isopter I. (A) Left eye before surgery: concentric narrowing of all isopters combined with focal temporal impairment of isopter I; (B) right eye before surgery: NAD; (C) left eye after surgery: NAD; (D) right eye after surgery: NAD. NAD, no abnormality detected.

patients with minor stroke with a score of $\leq 5$ at the time of entering the study according to the National Institutes of Health Stroke Scale. Postoperatively mean sensitivity was significantly better and mean defect was significantly lower in both the ipsilateral and the contralateral eye. In our data, the effect was more pronounced in isopter area III, which includes the periphery of the fundus. As we know from peripheral artery occlusive disease the periphery is the first region to show functional or morphological defects (diminished hair and nail growth, ulcers, necroses). In glaucoma, the intraocular pressure exceeds intraocular blood pressure. Typically, the nerves furthest from the entry of the optic nerve fail first because of their distance from the central blood supply to the eye; thus, vision loss due to glaucoma tends to start at the edges with the peripheral visual field, finally leading to tunnel vision. In case of impaired intraocular blood pressure, in principle, the same problem appears but without the fatal extent. In this situation, the intraocular blood pressure is not high enough to sufficiently supply the periphery of the fundus. So we conclude that revascularisation of the ICA leads to a better postoperative peripheral perfusion of the fundus.

In May 2008, a Russian group (Gavrilenko et $a l^{11}$ ) reported 180 patients with ocular ischaemic disorders (amaurosis fugax, occlusion of the central retinal artery and its branches, acute ischaemic optic neuropathy). After reconstructive surgery of the carotid artery, they found improved visual acuity, threshold of electrical sensitivity and electrical liability of the optical nerve. Although these results were obtained from symptomatic patients rather than a mixed population (asymptomatic and symptomatic) as in our study, they are in line with our outcome.

Telman $e t a l^{12}$ reported that with carotid stenosis, there is reversed flow in the ophthalmic artery in $15 \%$ of asymptomatic patients, and in $23 \%$ of symptomatic patients. In all cases, the blood flow reversed perioperatively and was orthograde postoperatively. This study demonstrates that impaired blood flow in the ophthalmic artery caused by internal carotid stenosis can be improved by internal carotid endarterectomy. Our study focused on functional aspects. We had six patients with preoperative visual field concentric narrowing. In three of those it disappeared completely postoperatively, in the other ones it improved remarkably. Our results with improved visual field are consistent with the findings by Telman $e t$ al of postoperative enhanced blood supply.

Not all patients had the same amount of visual field improvement and $10.3 \%(3 / 29)$ had focal impairments. Patients were not aware of these deteriorations thus these were 'silent' events. Kuliha $e t a l^{3}$ could show on MRI that new silent brain infarctions can be detected in up to $25 \%$ following ICE. The major part $(66 \%)$ in their study population was symptomatic with a modified Rankin score of 0-2 points, whereas our study included 


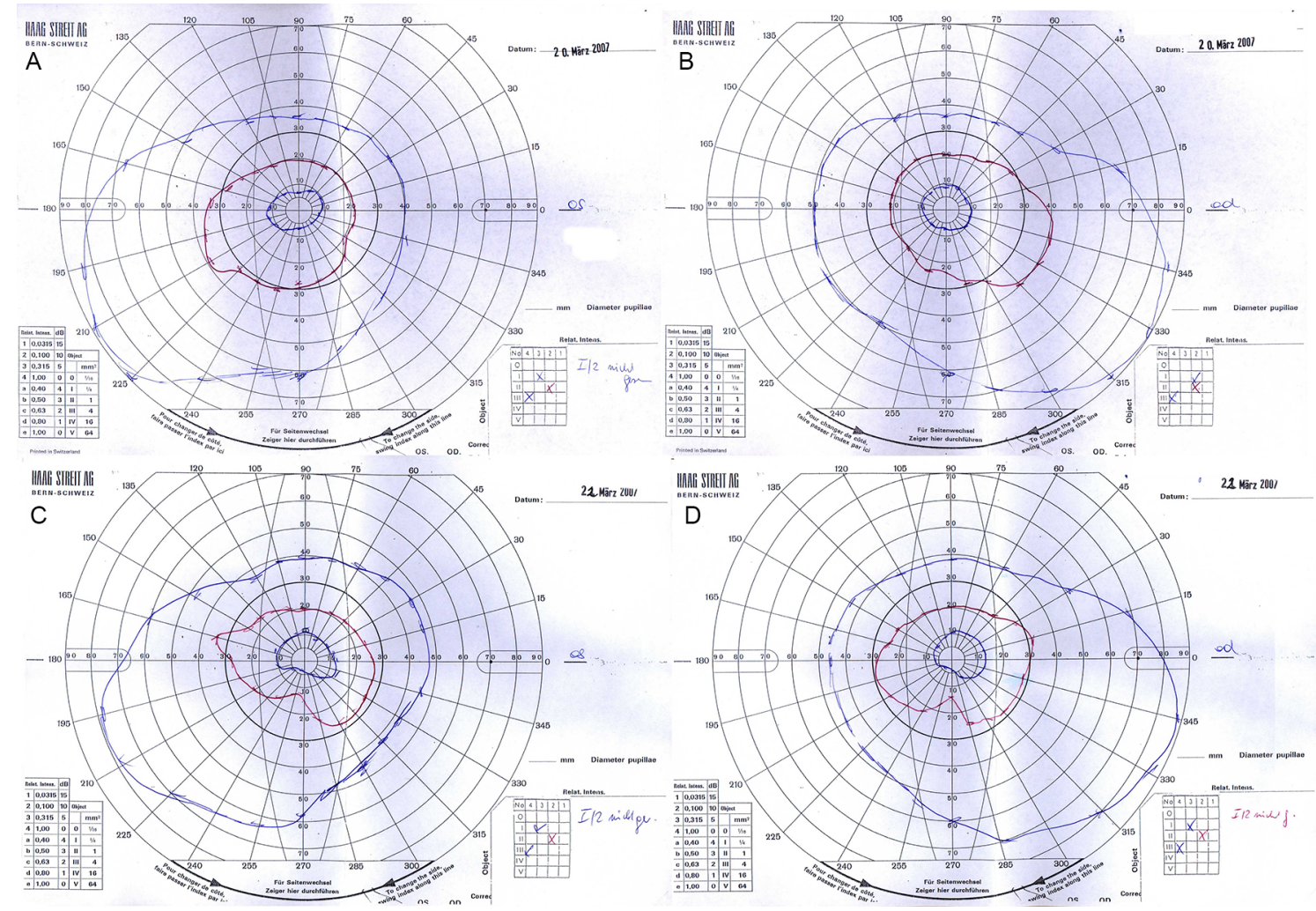

Figure 2 Preoperative and postoperative perimetry of patient \#2. Outer blue line: isopter III, red line: isopter II, inner blue line: isopter I. (A) Left eye before surgery: NAD; (B) right eye before surgery: NAD; (C) left eye after surgery: focal temporal impairment; (D) right eye after surgery: NAD. NAD, no abnormality detected.

$31 \%(9 / 29)$ symptomatic patients. Taking into account the different rate of symptomatic cases, their silent brain infarction rate and our silent visual field deterioration rate fit together very well. Analogously, we assume these focal impairments due to microembolisms.
Although there were some area differences according to different influencing factors (compare table 3), the study sample was too small to prove statistically significance. Corresponding to the study protocol the postoperative visual field examination was performed within 48 hours

Table 2 Isopter areas (given as ratio compared with the whole perimetric circle)

\begin{tabular}{|c|c|c|c|c|}
\hline & Preoperative & Postoperative & Difference & p Value \\
\hline Side of operation & $0.015(0.012$ to 0.020$)$ & $0.018(0.014$ to 0.023$)$ & $17.9 \%(0.0027)$ & 0.131 \\
\hline Difference & $11.6 \%(0.0018)$ & $13.8 \%(0.0025)$ & & \\
\hline$p$ Value & 0.227 & 0.099 & & \\
\hline Side of operation & 0.107 (0.094 to 0.122$)$ & $0.120(0.106$ to 0.134$)$ & $11.9 \%(0.0127)$ & 0.087 \\
\hline Contralateral side & $0.119(0.107$ to 0.132$)$ & 0.125 (0.114 to 0.137$)$ & $5.3 \%(0.0063)$ & 0.333 \\
\hline Difference & $11.2 \%(0.0120)$ & $4.7 \%(0.0057)$ & & \\
\hline $\mathrm{p}$ Value & 0.129 & 0.308 & & \\
\hline \multicolumn{5}{|l|}{ Isopter area III } \\
\hline Difference & $6.1 \%(0.0246)$ & $1.9 \%(0.0082)$ & & \\
\hline $\mathrm{p}$ Value & 0.290 & 0.492 & & \\
\hline
\end{tabular}




\begin{tabular}{|c|c|c|}
\hline & $\begin{array}{l}\text { Mean area } \\
\text { difference }\end{array}$ & p Value \\
\hline Contralateral ICA occlusion & & 0.386 \\
\hline No & 0.035 & \\
\hline Yes & 0.005 & \\
\hline $\begin{array}{l}\text { Grade of ipsilateral ICA stenosis } \\
\text { (NASCET) }\end{array}$ & & 0.238 \\
\hline $70 \%-80 \%$ & 0.017 & \\
\hline$>80 \%$ & 0.056 & \\
\hline Residual cerebral blood flow & & 0.756 \\
\hline Clinical status & & 0.326 \\
\hline Asymptomatic & 0.0221 & \\
\hline Symptomatic & 0.0467 & \\
\hline
\end{tabular}

ICA, internal carotid artery.

after surgery. Some patients were weak and had difficulties to concentrate, in particular those with visual field examination within 24 hours after surgery. Therefore, a postponement of this examination could have improved the postoperative results. The study protocol did not contain examinations after discharge from the hospital thus it is unclear, if the postoperatively determined visual field improvements are persistent in the long run.

The results are generalisable as far as the framework is similar. Our patients had surgery under general anaesthesia. In many hospitals, internal carotid endarterectomy is performed under local anaesthesia. These patients could be fitter 24 hours after surgery and reveal better results immediately after surgery. Another question is the extent of the internal carotid stenosis. If indication for surgery is based on internal carotid stenosis of $<70 \%$, blood flow improvement could be smaller than in our sample.

\section{CONCLUSIONS}

Internal carotid endarterectomy reduces the risk of cerebral embolism and is associated with visual field improvements. Further studies with more patients will enable us to identify subgroups with a high probability for visual field improvements to predict functional operative outcome. Another study is needed to assess the proportion of patients with carotid occlusive disease within those with concentric impairment.

Correction notice This article has been corrected since it was first published. Two proof-related annotations were removed from the final paper.

Acknowledgements The authors would like to thank Eugenia Lamont, Medical Editor at the Section for Surgical Research, Medical University of Graz, for critical review with particular attention to language.
Contributors PK (guarantor): study conception and design, analysis and interpretation of data, drafting of manuscript. IS: ophthalmologic data acquisition, critical revision of manuscript. SK: informed consent, surgical data acquisition, critical revision of manuscript. JM: informed consent, surgical data acquisition, critical revision of manuscript. AW: study conception and design, critical revision of manuscript. TC: study conception and design, critical revision of manuscript.

Competing interests None declared.

Ethics approval This study was submitted to, assessed and permitted by the Ethics Committee of the Medical University of Graz (28-437) in accordance with the principles of the Declaration of Helsinki and the ICH-GCP Guidelines while at the same time adhering to the relevant provisions of the Austrian Medicinal Products Act, the Austrian Medical Devices Act, the Styrian Hospitals Act, the Federal Hospitals and Health Spas Act and including all other relevant, eligible legislative provisions.

Provenance and peer review Not commissioned; externally peer reviewed.

Data sharing statement The relevant anonymised patient level data are available on reasonable request from the authors.

Open Access This is an Open Access article distributed in accordance with the Creative Commons Attribution Non Commercial (CC BY-NC 4.0) license, which permits others to distribute, remix, adapt, build upon this work non-commercially, and license their derivative works on different terms, provided the original work is properly cited and the use is non-commercial. See: http://creativecommons.org/ licenses/by-nc/4.0/

(C) Article author(s) (or their employer(s) unless otherwise stated in the text of the article) 2017. All rights reserved. No commercial use is permitted unless otherwise expressly granted.

\section{REFERENCES}

1. The CASANOVA Study Group. Carotid surgery versus medical therapy in asymptomatic carotid stenosis. Stroke 1991;22:1229-35.

2. Mayo Asymptomatic Carotid Endarterectomy Study Group. Results of a randomized controlled trial of carotid endarterectomy for asymptomatic carotid stenosis. Mayo Clin Proc 1992;67:513-8.

3. Hobson RW, Weiss DG, Fields WS, et al. Efficacy of carotid endarterectomy for asymptomatic carotid stenosis. N Engl J Med Overseas Ed 1993;328:221-7.

4. Executive Committee fort he Asymptomatic Carotid Atherosclerosis Study. Endarterectomy for asymptomaqtic carotid artery stenosis. $J$ Am Med Assoc 1995;273:1421-8.

5. Barnett HJM, Taylor DW, Haynes RB, et al. Beneficial effect of carotid endarterectomy in symptomatic patients with high-grade carotid stenosis. N Engl J Med 1991;325:445-53.

6. European Carotid Surgery Trialists' Collaborative Groupe. Randomised trial of endarterectomy for recently symptomatic carotid stenosis: final results of the MRC European Carotid Surgery Trial (ECST). Lancet 1998:351:1379-87.

7. Barnett HJM, Taylor DW, Eliasziw M, et al. Benefit of carotid endarterectomy in patients with symptomatic moderate or severe stenosis. N Engl J Med Overseas Ed 1998;339:1415-25.

8. Konstantiniuk P, Cohnert T. Acute monocular blindness after carotid endarterectomy due to non-arteritic anterior ischemic optic neuropathy. Eur J Vasc Endovasc Surg 2015;50:419.

9. Kozobolis VP, Detorakis ET, Georgiadis GS, et al. Perimetric and retrobulbar blood flow changes following carotid endarterectomy. Graefes Arch Clin Exp Ophthalmol 2007;245:1639-45.

10. Qu L, Feng J, Zou S, et al. Improved visual, acoustic, and neurocognitive functions after carotid endarterectomy in patients with minor stroke from severe carotid stenosis. J Vasc Surg 2015:62:635-44.

11. Gavrilenko !, Avetisov S, Kiseleva T, et al. Reconstructive surgery on the carotid arteries for ocular ischaemic disturbances correction. Interact Cardio Vasc Thorac Surg 2008;7:2.

12. Telman G, Kouperberg E, Nitecki S, et al. Cerebral hemodynamics in symptomatic and asymptomatic patients with severe unilateral carotid stenosis before and after carotid endarterectomy. Eur J Vasc Endovasc Surg 2006;32:375-8.

13. Kuliha M, Roubec M, Procházka V, et al. Randomized clinical trial comparing neurological outcomes after carotid endarterectomy or stenting. Br J Surg 2015;102:194-201. 